\title{
Eficacia antimicrobiana del aceite esencial de eucalipto (Eucalyptus spp) sobre cepas de Escherichia coli y Staphylococcus aureus subsp. aureus
}

\author{
Antimicrobial efficacy of eucalyptus essential oil (Eucalyptus spp) on Escherichia \\ coli and Staphylococcus aureus subsp. aureus strains \\ Mayra Montero-Recalde ${ }^{1,2}$, María José Morocho-Núñez ${ }^{1}$, Diana Avilés-Esquivel ${ }^{1}$, \\ Ángela Carrasco-Cando ${ }^{1}$, Ramiro Erazo-Gutierrez ${ }^{1}$
}

\section{Resumen}

El objetivo del estudio fue evaluar el efecto antimicrobiano in vitro del aceite esencial de eucalipto (Eucaliptus spp) sobre Escherichia coli ATCC ${ }^{\circledR} 11229$ y Staphylococcus aureus subsp. aureus ATCC $\AA^{\circledR} 25904$. Se evaluaron concentraciones al 30, 60 y $90 \%$ en dilución en etanol al 96.8\%. Se determinó la Concentración Mínima Inhibitoria (CMI) mediante el método de microdilución en caldo. El inóculo bacteriano se estandarizó al 0.5 de la escala de MacFarland en espectofotómetro, obteniendo como resultado que las concentraciones de 60 y $90 \%$ no presentaron turbidez. La Concentración Mínima Bactericida (CMB) determinada en agar Mueller-Hinton se presentó a partir de la concentración del $60 \%$ para las dos cepas en estudio. La prueba de sensibilidad antimicrobiana indicó que todas concentraciones presentaron sensibilidad antimicrobiana y que las concentraciones al 30 y $60 \%$ no fueron significativamente diferentes, presentando halos de inhibición de $10.25 \mathrm{~mm}$ y $10.65 \mathrm{~mm}$ para la concentración de $30 \%$ para las cepas de Escherichia coli y Staphylococcus aureus subsp. aureus, respectivamente. Por otro lado, los resultados para la cepa $S$. aureus subsp. aureus muestran que las concentraciones al $60 \%$ y $90 \%$ formaron halos de mayor diámetro que la cepa de $E$. coli.

Palabras clave: Concentración Mínima Inhibitoria; Concentración Mínima Bactericida; MacFarland

\footnotetext{
${ }^{1}$ Universidad Técnica Ambato, Campus Querochaca, Cevallos, Tungurahua, Ecuador

${ }^{2}$ E-mail: mayrismontero@yahoo.es
} 
The objective of this study was to evaluate the in vitro antimicrobial effect of eucalyptus essential oil (Eucaliptus spp) on Escherichia coli ATCC ${ }^{\circledR} 11229$ and Staphylococcus aureus subsp. aureus ATCC® 25904. Concentrations of 30, 60 and 90\% were evaluated in dilution in $96.8 \%$ ethanol. The Minimum Inhibitory Concentration (MIC) was determined by the broth microdilution method. The bacterial inoculum was standardized to 0.5 of the MacFarland scale in spectrophotometer, obtaining as a result that the concentrations of 60 and $90 \%$ did not present turbidity. The Minimum Bactericidal Concentration (MBC) determined in Mueller-Hinton agar was present from the $60 \%$ concentration for the two strains under study. The antimicrobial susceptibility test indicated that all concentrations showed antimicrobial sensitivity and that the concentrations at 30 and $60 \%$ were not significantly different, presenting halos of inhibition of $10.25 \mathrm{~mm}$ and $10.65 \mathrm{~mm}$ in the concentration of $30 \%$ for the strains of Escherichia coli and Staphylococcus. aureus subsp. aureus, respectively. On the other hand, the results for the strain S. aureus subsp. aureus showed that concentrations at $60 \%$ and $90 \%$ formed larger diameter halos than the $E$. coli strain.

Key words: Minimum Inhibitory Concentration; Minimum Bactericidal Concentration; MacFarland

\section{INTRODUCCIÓN}

El cultivo y uso de las plantas medicinales de los Andes ecuatorianos se ha expandido por Europa y el continente americano $(\mathrm{Ce}-$ rón, 2006). Entre los productos naturales, los aceites esenciales aromáticos y las plantas medicinales han recibido especial atención como posibles agentes naturales para la conservación de los alimentos (Burt, 2004). De hecho, se conoce que los aceites esenciales y sus componentes principales poseen un amplio espectro de actividad antimicrobiana (Gachkar et al., 2007).

La demanda de productos derivados de plantas para usos terapéuticos se ha incrementado en las últimas décadas (Hermann y von Richter, 2012). En las zonas rurales de varios países se utilizan las plantas aromáticas para la atención primaria de salud (Kamatou, 2005), siendo que el 80\% de las poblaciones rurales de países en desarrollo utilizan estos recursos tradicionales (Begossi, 1996). Por otro lado, el uso de acei- tes esenciales tiene fines clínicos de gran importancia en la investigación científica y la aplicación industrial por sus diversas actividades biológicas como antimicrobianos (Lo Cantore et al., 2009), antioxidantes (Dutra et al., 2008) y antiinflamatorios (Chao et al., 2005).

La resistencia cada vez mayor de las bacterias patógenas a los antimicrobianos plantea una grave amenaza para el tratamiento efectivo de un rango de infecciones causadas por estos microorganismos (Aslam et al., 2018). Con el aumento de la resistencia de patógenos a los antibacterianos sintéticos y la tendencia actual de los consumidores de no adquirir productos con este tipo de sustancias, se ha generado un gran interés en la investigación de los aceites esenciales como agentes antimicrobianos, así como por sus potenciales aplicaciones en los alimentos (Yáñez y Cuadro, 2012). Esto ha permitido que los aceites esenciales se presenten como buenos candidatos debido a compuestos presentes tales como terpenos y terpenoides $(1,8$ cineol, carvacrol) y compuestos aromáticos 
(cinamaldehído y eugenol) que poseen actividad antimicrobiana ante una amplia gama de patógenos y con diversos espectros de actividad (Bassolé y Juliani, 2012).

La actividad bactericida y antifúngica de estas sustancias está estrechamente relacionada con los fenoles y monoterpenos que poseen, debido a que son capaces de tener una interacción directa con el citoplasma del patógeno o bien, gracias a su hidrofobicidad, pueden incorporarse a los lípidos de la membrana celular bacteriana, en donde ocurre una fuga de iones y otros compuestos de la bacteria (El Asbahani, 2015). Los efectos antimicrobianos de los aceites esenciales están relacionados con su composición y efectos citotóxicos, que causan daño a la membrana celular (Bakkali et al., 2008).

El objetivo del proyecto de investigación fue evaluar el efecto antimicrobiano in vitro del aceite esencial de eucalipto (Eucaliptus spp) sobre Escherichia coli ATCC® 11229 y Staphylococcus aureus subsp. aureus ATCC® 25904.

\section{Materiales y Métodos}

\section{Ubicación Geográfica}

La investigación se realizó en el Laboratorio de Bacteriología de la Facultad de Ciencias Agropecuarias de la Universidad Técnica de Ambato, Campus Querochaca, ubicada en el cantón Cevallos, Tungurahua, Ecuador, a $2750 \mathrm{msnm}$. El estudio se realizó en un ambiente controlado a $20^{\circ} \mathrm{C}$ y humedad relativa de $48 \%$.

\section{Material Vegetal y Bacteriano}

Se utilizaron hojas frescas de Eucalipto (Eucalyptus spp) adquiridas en un mercado local de la ciudad de Ambato y las cepas certificadas de Escherichia coli ATCC ${ }^{\circledR} 11229$ y Staphylococcus aureus subsp. aureus $A T C C{ }^{\circledR} 25904$ de Medibac Ecuador (Corporate Headquarters, Minnesota, USA).
Se utilizaron las hojas más jóvenes del eucalipto, las cuales se lavaron en forma individual con agua bidestilada estéril y secadas en un lugar ventilado y oscuro por 24 horas. Luego fueron procesadas en el equipo tipo Clevenger, obteniéndose el aceite mediante el proceso de destilación por arrastre de vapor. Se prepararon concentraciones de $30,60,90(\mathrm{v} / \mathrm{v})$ de aceite esencial de eucalipto utilizando etanol al $96.8 \%$ como solvente. Se obtuvo $5 \mathrm{ml}$ de volumen total para cada concentración.

Las bacterias certificadas fueron activadas siguiendo las recomendaciones de la casa comercial, sembradas en cajas petri mediante la técnica de siembra escocés en Agar MacConkey y Agar Manitol Salado, respectivamente, e incubadas a $37^{\circ} \mathrm{C}$ por $24 \mathrm{~h}$. En la preparación del inóculo se utilizó el método del medio de cultivo líquido o de Kirby-Bauer, para lo cual se tomó en asa bacteriológica cinco colonias aisladas de cada una de las cepas certificadas y se las transfirió mediante un hisopo a $5 \mathrm{ml}$ de caldo cerebro corazón e incubadas a $37^{\circ} \mathrm{C}$ por 2 horas. Los inóculos fueron estandarizados en el espectrofotómetro con el fin de obtener un valor de 0.4 de absorbancia, donde la solución resultante tuvo $1 \times 10^{8}$ Unidades Formadoras de Colonias (UFC).

\section{Concentración Mínima Inhibitoria (CMI) y Concentración Mínima Bactericida (CMB)}

Para determinar la CMI se colocó $1 \mathrm{ml}$ del inóculo estandarizado en tubos de ensayo más $1 \mathrm{ml}$ de las concentraciones del aceite esencial de eucalipto e incubados a $37^{\circ} \mathrm{C}$ durante $24 \mathrm{~h}$. La menor turbidez fue establecida como la CMI, y determinada desde el $30 \%$ medida por espectofotómetro de $640 \mathrm{~nm}$ y $615 \mathrm{~nm}$ para Escherichia coli y Staphylococcus aureus subsp. aureus, respectivamente, a partir de inóculos estandarizados de $650 \mathrm{~nm}$ para ambas cepas bacterianas. 
Cuadro 1. Determinación de la concentración mínima inhibitoria (CMI) del aceite esencial de eucalipto (Eucalytus spp)

\begin{tabular}{lcccc}
\hline \multirow{2}{*}{ Cepas } & \multicolumn{3}{c}{$\begin{array}{c}\text { Diluciones del aceite esencial de } \\
\text { eucalipto }+ \text { etanol }\end{array}$} & $\begin{array}{c}\text { Control negativo } \\
+ \text { etanol } 96.8 \%\end{array}$ \\
\cline { 2 - 5 } & $30 \%$ & $60 \%$ & $90 \%$ & \\
\hline Escherichia coli & + & - & - & + \\
Staphylococcus aureus & + & - & - & + \\
\hline
\end{tabular}

,+- Turbidez

La CMB fue establecida al realizar la siembra de todos los tubos en agar MacConkey. La interpretación fue valorada a través del cero crecimiento de colonias en placa, la misma que fue a partir del $60 \%$ de la concentración del aceite esencial en las dos cepas en estudio.

\section{Sensibilidad Antimicrobiana}

Para la prueba de difusión por disco se tomaron cinco colonias de cada una de las cepas bacterianas (Escherichia coli y Staphylococcus aureus subsp. aureus) cultivadas previamente por $24 \mathrm{~h}$ en agar MacConkey y Manitol salado, respectivamente. Se inocularon en $5 \mathrm{ml}$ de caldo Cerebro-Corazón y se les incubó a $37{ }^{\circ} \mathrm{C}$ durante 2-3 h, hasta que apareció una turbidez semejante al tubo N. ${ }^{\circ} 0.5$ de la escala de MacFarland. La solución resultante tuvo $1 \times 10^{8}$ UFC.

Las cepas estandarizadas se cultivaron por estriado múltiple en agar Mueller Hinton, dejando reposar por $10 \mathrm{~min}$ antes de colocar los discos de sensibilidad Oxoid impregnados con las dosis del aceite esencial de eucalipto y etanol al $96.8 \%$ como testigo. Se impregnó una gota por cada disco de sensibilidad con las tres concentraciones $(30,60 \mathrm{y}$ $90 \%$ ) y se ubicaron cuatro discos de sensibilidad más el testigo en la superficie del agar de cada placa, repitiéndose por cuadruplicado por concentración y cepa bacteriana y se les llevó a incubar a $37^{\circ} \mathrm{C}$ durante 24 h. Posteriormente se midieron los halos de inhibición mediante el uso de la regleta $(\mathrm{mm})$.

\section{Resultados}

La interacción de las concentraciones del aceite de eucalipto y el crecimiento de las cepas Eschericia coli y Staphylococcus aureus mostraron un comportamiento definido a la razón de un incremento de la concentración y la turbidez reflejada (Cuadro 1). Los niveles de dilución del $30 \%$ en el caso de ambas cepas presentaron turbidez, refiriendo que existe un crecimiento bacteriano positivo, mientras que no hubo turbidez en las concentraciones del 60 y $90 \%$, indicando ausencia de crecimiento bacteriano.

La Concentración Mínima Bactericida (CMB), donde no existió crecimiento de colonias, se observó en las concentraciones del 60 y $90 \%$ del aceite de eucalipto para las dos cepas bacterianas, mientras que la concentración al 30\% del aceite presentó un desarrollo mínimo de colonias para ambas cepas (Cuadro 2).

En el Cuadro 3 se demuestra el efecto antimicrobiano del aceite esencial de eucalipto sobre ambas cepas bacterianas, siendo más específica para Staphylococcus aureus subsp. aureus a la concentración del 90\%, presentando un valor de $14.45 \mathrm{~mm}$, mientras 
Cuadro 2. Determinación de la concentración mínima bactericida (CMB) del aceite esencial de eucalipto (Eucalytus spp)

\begin{tabular}{|c|c|c|c|c|}
\hline \multirow[t]{2}{*}{ Cepas } & \multicolumn{3}{|c|}{$\begin{array}{l}\text { Tubos de ensayo con diluciones del } \\
\text { aceite esencial y cepas bacterianas } 1\end{array}$} & \multirow[t]{2}{*}{$\begin{array}{l}\text { Control negativo } \\
+ \text { etanol } 96.8 \% \\
\end{array}$} \\
\hline & $30 \%$ & $60 \%$ & $90 \%$ & \\
\hline Escherichia coli & + & - & - & + \\
\hline Staphylococcus aureus & + & - & - & + \\
\hline
\end{tabular}

,+- Crecimiento de colonias

${ }^{1} 1 \mathrm{ml}$ de cepa conteniendo $1.5 \times 108$ UFC y $1 \mathrm{ml}$ de cada concentración del aceite esencial

Cuadro 3. Halos de sensibilidad ( $\mathrm{mm}$ ) del aceite esencial de eucalipto (Eucalytus spp)

\begin{tabular}{lccccc}
\hline \multirow{2}{*}{ Cepas } & \multicolumn{3}{c}{ Tratamientos } & \multirow{2}{*}{ E.E. } & \multirow{2}{*}{ Valor de P } \\
\cline { 2 - 4 } & $30 \%$ & $60 \%$ & $90 \%$ & & \\
\hline E. coli & $10.25^{\mathrm{b}}$ & $10.65^{\mathrm{ab}}$ & $10.95^{\mathrm{a}}$ & 0.1230 & $<0.01$ \\
S. aureus & $10.95^{\mathrm{b}}$ & $13.65^{\mathrm{a}}$ & $14.45^{\mathrm{a}}$ & 0.4820 & $<0.001$ \\
\hline
\end{tabular}

${ }^{a, b}$ Medias con letras diferentes dentro de filas difieren significativamente

que la concentración de mayor eficacia para la cepa de Escherichia coli resultó ser del $90 \%$ con un valor de $10.95 \mathrm{~mm}$.

\section{Discusión}

El aceite de eucalipto mostró resultados positivos al inhibir in vitro el crecimiento de las cepas bacterianas Escherichia coli ATCC® 11229 y Staphylococcus aureus subsp. aureus ATCC ${ }^{\circledR} 25904$, presentándose como alternativa el empleo de productos naturales para reducir el uso indiscriminado de antibióticos (Cabrera et al., 2007; Reyes et al., 2012). Los aceites esenciales son el producto final de algunas plantas aromáticas, constituidos por terpenos con actividad antibacteriana, antimicótica, antiparasitaria, insecticida y antiviral (Alzamora et al., 2001).
En la presente investigación se utilizó el método de difusión en agar, al igual que en Yáñez y Cuadro (2012), demostrando la eficacia de este método para la evaluación de la actividad antimicrobiana de los aceites esenciales, pudiendo dar resultados cualitativos y cuantitativos (NCCLS, 2012).

El estudio permitió determinar la CMB del aceite esencial, correspondiendo al cese del crecimiento de colonias, como lo reporta Brochot et al. (2017). La CMI del aceite esencial de eucalipto se presentó a partir del $30 \%$, mientras que Yáñez y Cuadro (2012) obtuvieron CMI menores frente a bacterias grampositivas en comparación a gramnegativas, lo cual se debe a las características constitutivas de la pared celular, además de la concentración de los inóculos trabajados en las investigaciones. Por otro lado, Brochot 
et al. (2017) encontraron que la CMI se presentó a partir de una concentración de $0.38 \%$ para Staphylococcus aureus y de $0.78 \%$ para E. coli.

La prueba de sensibilidad antimicrobiana del aceite esencial de eucalipto mostró valores más eficaces para la bacteria grampositiva, al igual que Ait-Ouozzou et al. (2011), donde manifiestan que el aceite de eucalipto presentó efecto antibacteriano con predilección a bacterias grampositivas como Staphylococcus aureus. Por otro lado, Vieira et al. (2017) demostraron que el efecto antimicrobiano disminuye para cepas gramnegativas como E. coli.

La prueba de sensibilidad sobre Staphylococcus aureus subsp. aureus, en la presente investigación, reportó un valor máximo en su diámetro de halos de inhibición de $14.45 \mathrm{~mm}$ al $90 \%$, mientras que Elaissi et al. (2012) obtuvieron halos de $9 \mathrm{~mm}$. Así mismo, Ait-Ouazzou et al. (2011) reportaron halos de $14 \mathrm{~mm}$ como valor máximo. Por otro lado, $E$. coli mostró un valor máximo en su diámetro de halos de inhibición de $10.95 \mathrm{~mm}$ al 90\%, mientras que Vieira et al., (2017) obtuvieron un diámetro máximo de halos de sensibilidad de $9 \mathrm{~mm}$ con el aceite sin dilución. Así mismo, Ait-Ouazzou et al. (2011) no obtuvieron resultados positivos con el aceite de eucalipto (Eucalyptus globulus) sobre la cepa de E. coli.

\section{Conclusiones}

- El aceite esencial de eucalipto (Eucaliptus spp) es más efectivo sobre la cepa Staphylococcus aureus subsp. aureus (grampositiva) que sobre la cepa de Escherichia coli (gramnegativa).

- La Concentración Mínima Inhibitoria (CMI) del aceite esencial de eucalipto sobre ambas cepas fue a partir del $30 \%$.

- La Concentración Mínima Bactericida (CMB) fue a partir del $60 \%$ para ambas cepas.
- La cepa Staphylococcus aureus subsp. aureus presentó mayor sensibilidad al aceite esencial de eucalipto que la cepa Escherichia coli, en referencia al diámetro de los halos de sensibilidad.

\section{Literatura Citada}

1. Ait-Ouazzou A1, Lorán S, Bakkali M, Laglaoui A, Rota C, Herrera A, Pagán $\boldsymbol{R}$, et al. 2011. Chemical composition and antimicrobial activity of essential oils of Thymus algeriensis, Eucalyptus globulus and Rosmarinus officinalis from Morocco. J Sci Food Agr 91: 2643 2651. doi: $10.1002 /$ jsfa. 4505

2. Alzamora L, Morales L, Armas L, Fernández G. 2001. Medicina tradicional en el Perú: actividad antimicrobiana in vitro de los aceites esenciales extraídos de algunas plantas aromáticas. Anales Fac Med 62: 156-161. doi: 10.15381/ anales.v62i2.4167

3. Aslam B, Wang W, Imran Arshad M, Khurshid M, Muzammil S, Hidayat Rasool M, Atif Nisar M, et al. 2018. Antibiotic resistance: a rundown of a global crisis. Infect Drug Resist 2018:11 1645-1658. doi: 10.2147/idr.s173867

4. Bakkali F, Averbeck S, Averbeck D, Idaomar M. 2008. Biological effects of essential oils - a review. Food Chem Toxicol 46: 446-475. doi: 10.1016/ j.fct.2007.09.106

5. Bassolé IH, Juliani HR. 2012. Essential oils in combination and their antimicrobial properties. Molecules 17: 3989-4006. doi: 10.3390/molecules17043989

6. Begossi A. 1996. Use of ecological methods in ethnobotany: diversity indices. Econ Bot 50: 280-289. doi: 10.1007/ BF02907333

7. Brochot A, Guilbot A, Haddioui L, Roques $C$. 2017. Antibacterial, antifungal, and antiviral effects of three essential oil blends. Microbiologyopen 6: e00459. doi: 10.1002/mbo3.459 
8. Burt S. 2004. Essential oils: their antibacterial properties and potential applications in foods - a review. Int $\mathrm{J}$ Food Microbiol 94: 223-253. doi: 10.1016/ j.ijfoodmicro.2004.03.022

9. Cabrera CE, Gomez RF, Zúñiga AE. 2007. La resistencia de bacterias a antibióticos, antisépticos y desinfectantes una manifestación de los mecanismos de supervivencia y adaptación. Colombia Médica 38: 149-158.

10. Cerón CE. 2006. Plantas medicinales de los Andes ecuatorianos. Botánica Econ Andes Centrales 2006: 285-293.

11. Chao LK, Hua KF, Hsu HY, Cheng SS, Liu JY, Chang ST. 2005. Study on the antiinflammatory activity of essential oil from leaves of Cinnamomum osmophloeum. J Agr Food Chem 53: 7274-7278. doi: 10.1021/jf051151u

12. Dutra RC, Leite MN, Barbosa NR. 2008. Quantification of phenolic constituents and antioxidant activity of Pterodon emarginatus vogel seeds. Int J Mol Sci 9: 606-614. doi: 10.3390/ ijms9040606

13. Elaissi A, Rouis Z, Mabrouk S, Salah KB, Aouni M, Khouja ML, Farhat F, et al. 2012. Correlation between chemical composition and antibacterial activity of essential oils from fifteen Eucalyptus species growing in the Korbous and Jbel Abderrahman arboreta (North East Tunisia). Molecules 17: 3044-3057. doi: 10.3390/ molecules17033044

14. El Asbahani A, Miladi K, Badri W, Sala M, Addi EA, Casabianca $\mathrm{H}, \mathrm{El}$ Mousadik A, et al. 2015. Essential oils: from extraction to encapsulation. Int $\mathrm{J}$ Pharm 483: 220-243. doi: 10.1016/ j.ijpharm.2014.12.069

15. Gachkar L, Yadegari D, Bagher Rezaei M, Taghizadeh M, Alipoor Astaneh S, Rasooli I. 2007. Chemical and biological characteristics of Cuminum cyminum and Rosmarinus officinalis essential oils. Food Chem 102: 898-904. doi: 10.1016/j.foodchem.2006.06.035

16. Hermann R, von Richter O. 2012. Clinical evidence of herbal drugs as perpetrators of pharmacokinetic drug interactions. Planta Med 78: 1458-1477. doi: $10.1055 / \mathrm{s}-0032-1315117$

17. Kamatou GP, Viljoen AM, GonoBwalya $A B$, van $Z y l R L$, van Vuuren $S F$, Lourens AC, Baser KH, et al. 2005. The in vitro pharmacological activities and a chemical investigation of three South African Salvia species. J Ethnopharmacol 102: 382-390. doi: 10.1016/j.jep.2005.06.034

18. Lo Cantore P, Shanmugaiah V, Sante N. 2009. Antibacterial activity of essential oil components and their potential use in seed disinfection. J Agr Food Chem 57: 9454-9461. doi: 10.1021/ j1902333g

19. [NCCLS] National Committee for Clinical Laboratory Standars. 2012. Performance Standards for Antimicrobial Disk Susceptibility Test. M02-A11.NCCLS $32.76 \mathrm{p}$.

20. Reyes-Jurado F, Palou E, López-Malo A. 2012. Vapores de aceites esenciales: alternativa de antimicrobianos naturales. Temas Selectos de Ingeniería de Alimentos 6: 29-39.

21. Vieira M, Bessa LJ, Martins MR, Arantes S, Teixeira APS, Mendes A, Martins da Costa P, et al. 2017. Chemical composition, antibacterial, antibiofilm and synergistic properties of essential oils from Eucalyptus globulus Labill. and seven Mediterranean aromatic plants. Chem Biodivers 14: e1700006. doi: 10.1002/cbdv.201700006

22. Yáñez X, Cuadro OF. 2012. Composición química y actividad antibacteriana del aceite esencial de las especies Eucalyptus globulus y E. camaldulensis de tres zonas de Pamplona (Colombia). Bistua 10: 52-61. 